\title{
Energy dissipation of atomic-scale friction based on one- dimensional Prandtl-Tomlinson model
}

\author{
Zi-Jian WANG ${ }^{1}$, Tian-Bao MA ${ }^{1, *}$, Yuan-Zhong HU ${ }^{1}$, Liang XU' ${ }^{2}$, Hui WANG ${ }^{1}$ \\ ${ }^{1}$ State Key Laboratory of Tribology, Tsinghua University, Beijing 100084, China \\ ${ }^{2}$ Beijing Institute of Nanoenergy and Nanosystems, Chinese Academy of Sciences, Beijing 100083, China \\ Received: 27 March 2015 / Revised: 16 May 2015 / Accepted: 01 June 2015 \\ (C) The author(s) 2015. This article is published with open access at Springerlink.com
}

\begin{abstract}
The energy transition and dissipation of atomic-scale friction are investigated using the one-dimensional Prandtl-Tomlinson model. A systematic study of the factors influencing the energy dissipation is conducted, indicating that the energy that accumulated during the stick stage does not always dissipate completely during stick-slip motion. We adopt the energy-dissipation ratio (EDR) to describe the relationship between the energy dissipated permanently in the system and the conservative reversible energy that can be reintroduced to the driving system after the slip process. The EDR can change continuously from $100 \%$ to 0 , covering the stick-slip, intermediate, and smooth-sliding regimes, depending on various factors such as the stiffness, potential-energy corrugation, damping coefficient, sliding velocity, and the temperature of the system. Among these, the parameter $\eta$, which depends on both the surface potential and the lateral stiffness, is proven in this paper to have the most significant impact on the EDR. According to $\eta-T$ phase diagrams of the EDR, the smooth-sliding superlubricity and thermolubricity are found to be unified with regard to the energy dissipation and transition. An analytical formulation for the EDR that can be used to quantitatively predict the amount of energy dissipation is derived from a lateral-force curve.
\end{abstract}

Keywords: atomic-scale friction; energy reversibility; energy dissipation ratio; superlubricity

\section{Introduction}

Friction is mechanical kinetic-energy loss or the transformation of sliding motion into heat and other excitations [1-3]. Although atomic-scale frictional behavior and its influencing factors have been studied extensively, the process and amount of energy transfer and dissipation during stick-slip friction-which are essential for an in-depth understanding of atomic-scale friction-are rarely quantified. The elastic energy that accumulates during the stick stage can be dissipated irreversibly by heat generation during the slip stage [4-6]. Berman and Israelachvili postulated that in the cobblestone model, upon each molecular collision during sliding, only part of the kinetic energy is

*Corresponding author: Tian-Bao MA.

E-mail: mtb@mail.tsinghua.edu.cn dissipated, and the rest is reflected back to the system [7]. There have been experimental studies wherein a transition from a highly dissipative stick-slip motion to continuous sliding was observed with a gradual decrease in the friction [8], indirectly supporting this hypothesis, suggesting that there exist some intermediate states rather than an abrupt transition between the stick-slip and frictionless sliding. In theoretical studies, Rozman et al. attempted to divide the frictional force into the potential and dissipative components, where the friction can be viewed as a reversible, adiabatic process with a vanishing dissipative contribution in a quasi-static state [9]. However, the quantitative estimation of the amount of energy dissipation remains a challenge. The fraction of energy that dissipates during sliding is dependent on both the intrinsic system properties and influencing factors such as the sliding velocity and the temperature, which is the 
most intricate parameter and the most important to determine [7].

In this paper, the energy transition and dissipation under stick-slip friction are quantitatively examined using the Prandtl-Tomlinson (PT) model [10-12]. Factors influencing the energy dissipation, such as the stiffness, potential-energy corrugation, damping coefficient, sliding velocity, and temperature of the system, are systematically investigated. We find that the stiffness not only affects the energy entering the system but also, more importantly, affects the energy that can flow back to the driven system after a slip. Two mechanisms of superlubricity [13-15] - smooth sliding and thermolubricity-are discussed in the context of the energy dissipation. The formulation of the energy-dissipation ratio (EDR) is derived analytically to characterize the ratio between the dissipative energy and the total energy that accumulates during sticking.

\section{Simulation methodology}

All the simulations are based on the one-dimensional (1D) Prandtl-Tomlinson model with the assumption of an oscillator having a mass of $m\left(10^{-12} \mathrm{~kg}\right)$ sliding over a sinusoidal potential with amplitude $U(0.01 \mathrm{eV}<$ $U<1 \mathrm{eV})$ and periodicity $a(3 \AA)$. The oscillator is connected to a driving support with a constant speed $v_{\mathrm{RD}}$ by a harmonic spring with stiffness $k(1 \mathrm{~N} / \mathrm{m}<k<$ $100 \mathrm{~N} / \mathrm{m}$ ), as shown in Fig. 1. The PT model is a classical model for describing phenomena ranging from macro rigid mechanics to atomic-scale friction. There have been several important advances in the extension of the PT model. The Frenkel-Kontorova (FK) model $[16,17]$ employs a $1 D$ chain of atoms connected by springs, instead of a single oscillator. The FrenkelKontorova-Tomlinson (FKT) model [18] considers the size of contact. In the composite-oscillator model [19], to better describe the thermal lattice vibration, a macroscopic oscillator having a low frequency is coupled with micro oscillators having a high frequency. Nevertheless, presently, the 1D PT model is widely employed to examine the friction on both the macroscopic and microscopic scales. This reduced-order, atomic-scale friction model is particularly suitable to describe the atomic force microscopy (AFM) tip-sample interaction [20], which simplifies the single-asperity

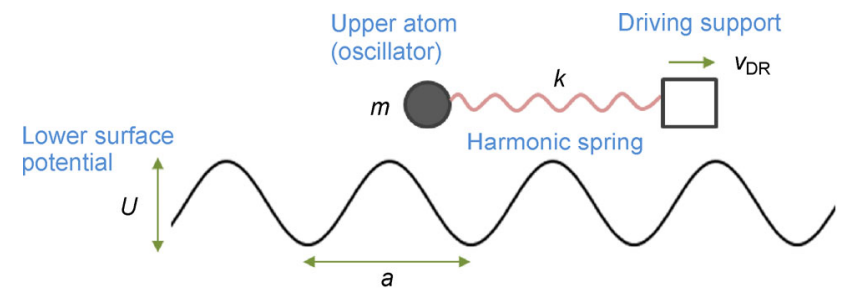

Fig. 1 Schematic of the 1D PT model. The upper surface is represented as an oscillator, and the lower surface is represented as a potential field with corrugation $U$ and period $a$.

friction into one point-mass (oscillator) pulled along the periodic lattice (potential energy profile) by an elastic cantilever (spring).

The dynamics of the system are solved using the Langevin equation [21, 22], which is shown in Eq. (1), with the fourth-order Runge-Kutta algorithm and a time step of $\Delta t=100 \mathrm{~ns}$ to achieve a high precision.

$$
m \ddot{x}+m \mu \dot{x}=-\frac{\partial P(x, t)}{\partial x}+\varepsilon(t)
$$

Here, $x$ is the coordinate of the oscillator along the sliding direction, $P$ is the potential energy in the system (including both the elastic and surface potential), and $\varepsilon(t)$ refers to the stochastic thermal-activation force. A system temperature of $0 \mathrm{~K}$ is used throughout this paper, except for Section 3.4. $\mu$ denotes the damping coefficient, which is calculated using Eq. (2), where $c(0.6<c<2.0)$ is a dimensionless parameter, and $\mu_{\mathrm{c}}=2 \sqrt{\mathrm{k} / \mathrm{m}}$ is the critical resonance frequency of the system [23].

$$
\mu=c \cdot \mu_{\mathrm{c}}=c \cdot 2 \sqrt{\frac{k}{m}}
$$

The driving support moves with a constant velocity of $v_{\mathrm{DR}}=1 \mu \mathrm{m} / \mathrm{s}$; thus, at every instant, the elongation of the spring is $\left(v_{D R} \cdot t-x(t)\right)$, where $x(t)$ is the displacement of the oscillator at the moment $t$. During an entire stick-slip period, the system energy can be divided into four forms: the surface potential $\left(P_{\mathrm{s}}\right)$, elastic potential of the spring $\left(P_{\mathrm{e}}\right)$, kinetic energy of the oscillator $(K)$, and dissipation energy $(D)$. The surface potential is modeled in the sinusoidal form for simplicity. The spring connecting the oscillator and the driving support is harmonic, following Hooke's law. Thus, the elastic potential and surface potential are calculated using the following formulas: 


$$
\begin{gathered}
P_{\mathrm{e}}(t)=\frac{1}{2} k \cdot\left[v_{\mathrm{DR}} \cdot t-x(t)\right]^{2} \\
P_{\mathrm{s}}(t)=\frac{1}{2} U \cdot\left[1+\cos \left(\frac{2 \pi x(t)}{a}+\pi\right)\right]
\end{gathered}
$$

where the system potential energy consists of two parts: the elastic potential energy $\left(P_{\mathrm{e}}\right)$ and surface potential energy $\left(P_{\mathrm{s}}\right)$.

$$
P(t)=P_{\mathrm{e}}(t)+P_{\mathrm{s}}(t)
$$

The total system energy $\left(E_{\text {total }}\right)$, including the dissipation energy, is then calculated as follows:

$$
E_{\text {total }}(t)=P(t)+K(t)+D(t)
$$

Both the kinetic energy $K(t)$ and dissipation energy $D(t)$ are related to the velocity of the oscillator, $\dot{x}(t)$. Here, the dissipation energy is calculated by summing the work done by the viscous damping forces, i.e., by performing a time integration of the damping force, as shown in Eq. (8). The damping coefficient is a comprehensive, system-dependent quantity that is affected by several factors. Phononic damping, electronic damping, and viscous damping can all contribute to the damping coefficient [3]. However, in the present paper, the damping force is calculated as $f_{\mathrm{D}}=m \mu \dot{x}$, which is a widely accepted approximation [23-25]. Distinguishing the exact channel of the frictionalenergy dissipation remains a challenge and is required for a more accurate description of the damping coefficient. This issue is worth studying further in future works.

$$
\begin{array}{r}
K(t)=\frac{1}{2} m \cdot \dot{x}^{2} \\
D(t)=\int_{0}^{x(t)} f_{\mathrm{D}} \mathrm{d} x
\end{array}
$$

The source of the energy, which is the work done by the external driving support $(W)$, is calculated as follows:

$$
W(t)=\int_{0}^{t} F \cdot v_{\mathrm{DR}} \mathrm{d} t
$$

The driving support moves uniformly. Thus, the lateral force $F$ should be always equal to the spring force, as indicated by Eq. (10):

$$
F=k\left[v_{\mathrm{DR}} \cdot t-x(t)\right]
$$

\section{Results and discussions}

\subsection{Energy transitions and reversibility}

The energy transitions during a complete stick-slip period are shown in Fig. 2. Most of the energy dissipation occurs during the slipping. Therefore, in this paper, we focus particularly on the slip stage. To clearly illustrate the energy transitions, a complete stick-slip

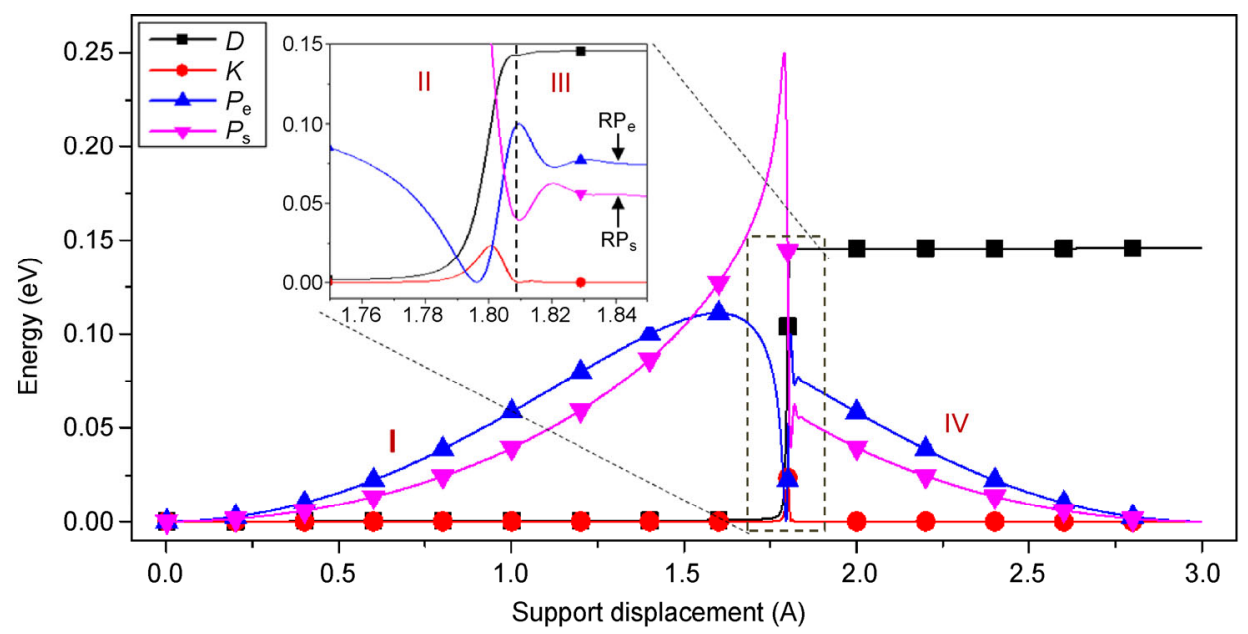

Fig. 2 Transition among different energy forms in a stick-slip period. In the stick stage (I), the surface potential energy and elastic energy accumulate, and a sudden drop occurs during the slip (stages II and III). After the slip, part of the potential energy remains, i.e., RP $\mathrm{e}_{\mathrm{e}}$ and $\mathrm{RP}_{\mathrm{s}}$, which is slowly released to the external system until the end of stage IV. The simulation is conducted under the conditions of $U=$ $0.25 \mathrm{eV}, k=5 \mathrm{~N} / \mathrm{m}$, and $c=0.6$. 
period can be divided into four stages, the first of which is the accumulation of potential energy (hereinafter called stage I). In this stage, the oscillator is pulled by the driving support, and potential energy accumulates gradually. When the energy accumulates to a certain extent, a slip takes place, which comprises two stages (II and III). Stage II is the major energy dissipation, i.e., the transformation of part of the potential energy into non-conservative energy. The sudden decreases in $P_{\mathrm{e}}$ and $P_{\mathrm{s}}$ and the increase in $K$ and $D$ occur synchronously. Stage III is the transformation of the residual energy. The potential energy and kinetic energy convert into each other during the oscillation. After the slip stage, the potential energy is not completely dissipated, and the reversible part is released to the external system. As shown in the inset of Fig. 2, at the end of stage III, $0.08 \mathrm{eV}$ of $P_{\mathrm{e}}$ and $0.05 \mathrm{eV}$ of $P_{\mathrm{s}}$ i.e., $\mathrm{RP}_{\mathrm{e}}$ and $\mathrm{RP}_{\mathrm{s}}$, respectively, remain. $R P_{e}$ and $R P_{s}$ constitute the reversible potential energy $(\mathrm{RP})$ :

$$
R P=R P_{e}+R P_{s}
$$

Stage IV involves the release of reversible energy and the onset of the next stick-slip. Here, the potential energy of the system decreases, but there is no change in the dissipation or kinetic energy, indicating that the residual energy is gradually transferred to the external system.

Figure 3(a) demonstrates the variation of the total system energy $\left(E_{\text {total }}\right)$ and external work $(W)$ during sliding, as indicated by Eqs. (6) and (9), respectively. The overlapping of the external work and total energy curves shows that all the external work of the driving support is used only to counteract the energy dissipation and maintain sliding. As presented in Fig. 3(a), the total energy accumulates during the stick stage, corresponding to the positive work performed by the driving support. Upon a slip, the total energy decreases, and negative work is performed by the driving support, indicating that not all the energy that accumulates during the stick dissipates permanently. Rather, the external system can retrieve part of the energy. The peak value $W_{1}$ is the total energy that accumulates in the stick stage, and $W_{2}$ represents the reversible energy (or work) transferred back to the external system. Thus, $W_{1}-W_{2}$ represents the energy dissipated during one stick-slip period. As shown in Fig. 2, the reversible energy $W_{2}$ comes from the potential energy $R P_{e}$ and $R P_{s}$ at the beginning of stage IV. Figure 3(b) shows the variation of the system potential energy $P$ during the stick-slip motion. Part of the potential energy is converted into dissipation energy (DP), and the remainder is reversible energy (RP).

To measure the degree of energy dissipation or energy reversibility, we present two definitions for the EDR: $\mathrm{EDR}_{W}$ and $\mathrm{EDR}_{P}$, which are described in Eqs. (12) and (13), respectively. Equation 12, derived from Fig. 3(a), is used to calculate the fraction of dissipated external work (W), i.e., EDR $\mathrm{ER}_{W}$. Equation 13, deduced from Fig. 3(b), is used to calculate the fraction of reversible potential energy during the slip stage, i.e., $\mathrm{EDR}_{P}$. These two definitions for the EDR are equivalent under the assumption that most of the
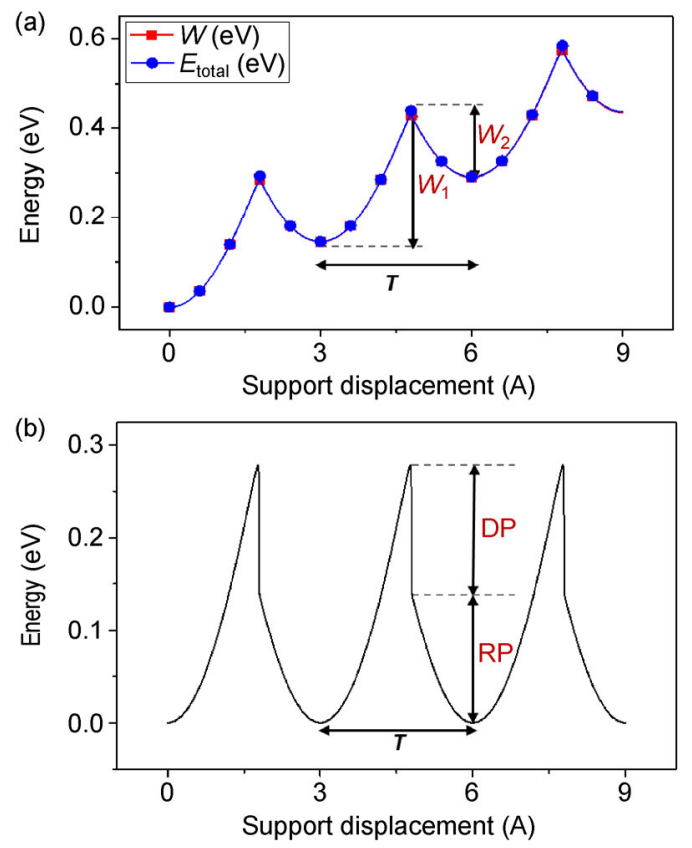

Fig. 3 Variations of system energy in stick-slip motion. (a) Variation of external work and total system energy during sliding. The peak value $W_{1}$ is the total energy that accumulates in the stick stage, and $W_{2}$ is the reversible energy (or work) transferred back to the external system. Thus, $\left(W_{1}-W_{2}\right)$ is the energy dissipated during one stick-slip period. (b) Variation of the system potential energy $P$ during the stick-slip motion. Part of the potential energy is converted into dissipation energy (DP), and the remainder is reversible energy (RP) that is transferred to the external system. The whole stick-slip period is denoted by $T$. The simulation is performed under the conditions indicated in Fig. 2. 
energy dissipation occurs at the slip stage because of the system instability, which applies to most tribological systems. However, the two definitions yield different EDR results under smooth sliding or similar conditions with a very small energy dissipation, where the dissipation due to the slip instability is negligibly small compared with the contribution of the viscous dissipation, which is proportional to the driving speed. In this paper, we investigate the energy dissipation caused by the stick-slip instability, which is better described by $\mathrm{EDR}_{P}$, i.e., the proportion of the irreversible potential energy during slipping to the total potential energy that accumulates in the stick stage. Therefore, unless otherwise specified, in the following calculations and discussions, $\mathrm{EDR}_{P}$ is applied as the definition of the EDR.

$$
\begin{aligned}
& \mathrm{EDR}_{W}=\frac{W_{1}-W_{2}}{W_{1}}=\frac{D}{W_{1}} \\
& \mathrm{EDR}_{P}=\frac{\mathrm{DP}}{P}=\frac{\mathrm{DP}}{\mathrm{DP}+\mathrm{RP}}
\end{aligned}
$$

What causes the energy reversibility in our system? To answer this question, the variation of the lateral force and the potential energy during the stick-slip are depicted in Fig. 4. In a complete period (denoted as " $T$ "), both the potential energy and lateral force vary in a periodic manner. The potential energy is slowly released after its suddenly decrease at the slip stage as the lateral force changes from positive to negative when the spring stiffness is $3 \mathrm{~N} / \mathrm{m}$, as shown in Fig. 4(a). However, when the spring stiffness is $1 \mathrm{~N} / \mathrm{m}$, the stage of the potential release is absent, and the potential increases gradually after the rapid decrease in the slip stage. In this case, no negative lateral force appears, as shown in Fig. 4(b). We believe that a negative lateral force is necessary for part of the energy to be reversible, because a negative lateral force indicates that part of the internal energy of the system can be transferred back to the external driver. The emergence of the negative lateral force is essentially caused by the over-slipping of the oscillator when the oscillator slips over the driving support, i.e., $x(t)>$ $v_{\mathrm{DR}} \cdot t$. According to Eq. (9), this results in negative work, thereby leading to energy reversibility.

\subsection{Controlling factors of EDR}

According to Lyapunov's criterion for stability [26, 27], the dimensionless parameter $\eta$ shown in Eq. (14) is appropriate to describe the system stability:

$$
\eta=\frac{2 \pi^{2} U}{k a^{2}}
$$
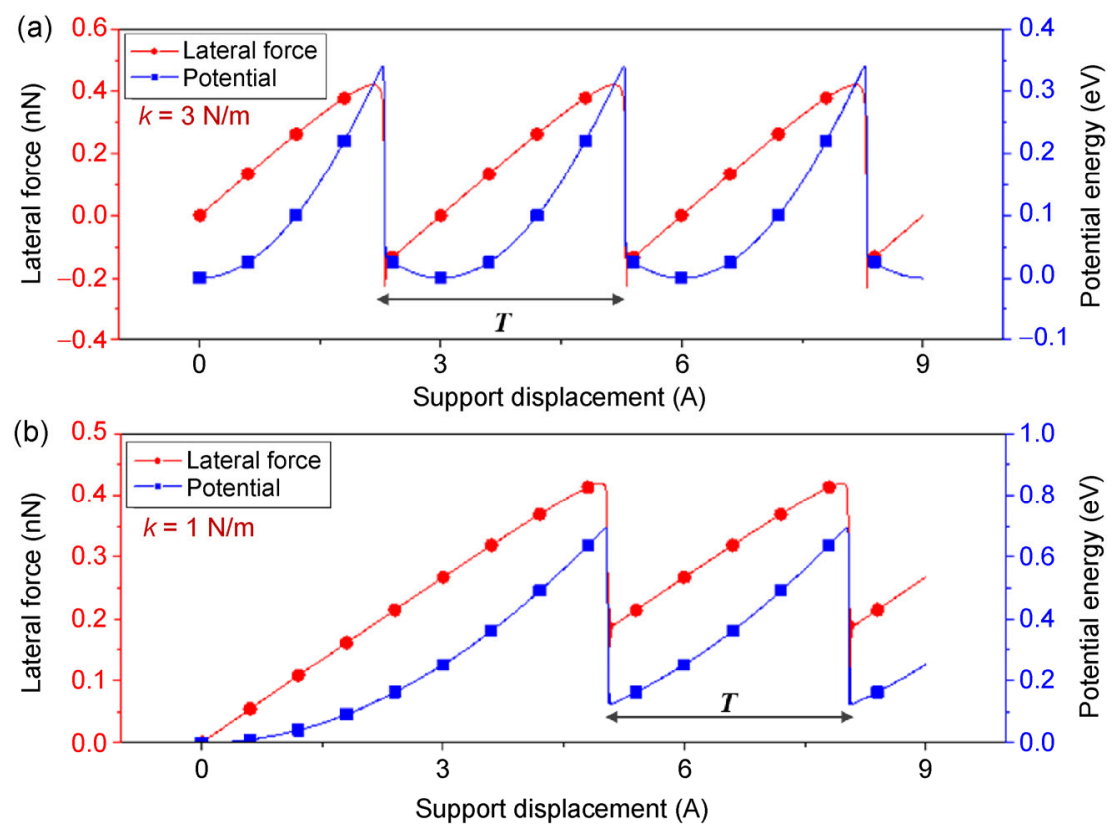

Fig. 4 Lateral-force and potential-energy curves with spring stiffness of (a) $k=3 \mathrm{~N} / \mathrm{m}$; (b) $k=1 \mathrm{~N} / \mathrm{m}$. Other parameters: $U=0.25 \mathrm{eV}, c=1$. 
This parameter is dependent on $U, k$, and $a$. If $\eta \geqslant 1$, stick-slip occurs, and if $\eta<1$, there is continuous sliding. $\eta$ is also a key parameter to differentiate stick-slip regimes $[8,28-30]$. It has been successfully used to predict atomic-scale friction behavior. Could it also describe the energy dissipation, specifically the EDR?

As shown in Fig. 5, a complete transition of the EDR from 0 to $100 \%$ is obtained by varying $\eta$, indicating that the transition from smooth sliding to stick-slip is a gradual process rather than a sudden change. For comparison, the curve is divided into three zones. In zone I, $\eta$ is smaller than 1.0, and the EDR is 0, which corresponds to a complete energy reversion or a perfect smooth-sliding regime. It should be mentioned that this does not suggest zero-energy dissipation, as the viscous friction due to the continuous sliding produces a very small amount of dissipation. The intermediate state (zone II), where the EDR value varies from 0 to $100 \%$, is the most common state in atomic-scale friction. In this regime, the system becomes instable, and stick-slip motion occurs. However, regarding the energy dissipation, there remains reversible energy in this regime. Thus, we named it the "intermediate regime" to distinguish it from zone III, where the EDR is $100 \%$. Instead of an abrupt change, the transition from the frictionless sliding to stick-slip is gradual and continuous, as shown in Fig. 5. Thus, there are only quantitativerather than qualitative-differences in the energy dissipation among the different intermediate states. Achieving the precise control of the system parameters

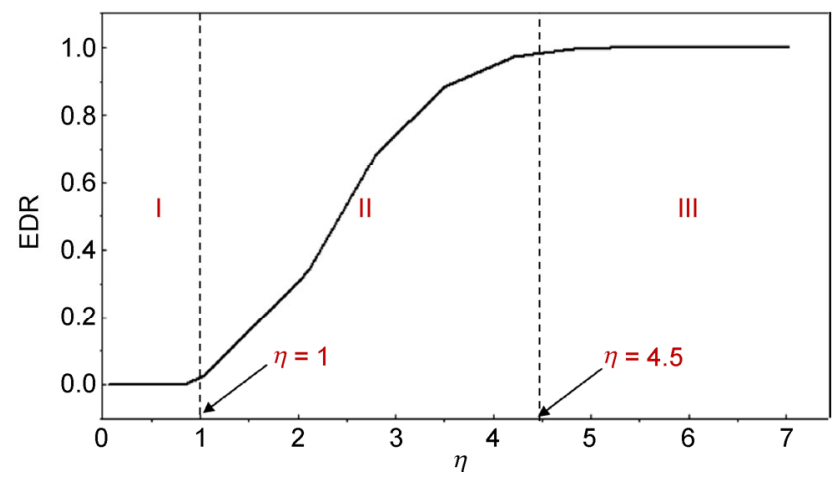

Fig. 5 EDR curve corresponding to various $\eta$ values. Regions I, II, and III indicate the smooth-sliding $(\mathrm{EDR}=0)$, intermediate $(0<\mathrm{EDR}<100 \%)$, and stick-slip regimes $(\mathrm{EDR}=100 \%)$, respectively. $\eta$ is varied by changing the spring stiffness $k$. is promising for controlling the states of atomic-scale friction. In zone III, where $\eta$ is sufficiently large, the EDR equals 100\%; thus, this can be called the stick-slip regime. The critical value of $\eta$ between the smoothsliding and intermediate regimes is estimated to be 1.0, and that between the intermediate and stick-slip regimes is 4.5, as shown in Fig. 5.

Notably, $\eta$ has a crucial impact on the slip style (e.g., single or multiple slips) [6,30], which affects the energy dissipation but is not the focus of this paper. To eliminate the influence of the slip style, calculations are conducted with a spring stiffness ranging from 0.1 to $100 \mathrm{~N} / \mathrm{m}$. This stiffness range ensures a single slip and is wide enough to cover both the stick-slip and smooth-sliding regimes. The surface potential corrugation $U$ and spring stiffness $k$ are equivalent for determining the EDR. This equivalency is also clearly indicated by Eq. (14). The parameter $\eta$ is used in our discussions, although the simulations presented in this section are based on a varying stiffness with a constant $U$ value, unless otherwise specified, for simplicity. The aforementioned result demonstrates that higher spring stiffness can contribute to a lower EDR; i.e., a stiffer spring improves the energy reversibility. This dependency is verified by our previous molecular-dynamics simulation, where the interlayer friction of few-layer graphene is found to be dependent on the number of layers. When the number of layers is decreased below three, the lateral stiffness of the system is sufficiently large, and the stick-slip phenomenon disappears, causing the friction to vanish [31].

To understand the effects of the spring stiffness, we first recall that the EDR indicates the ratio between the dissipative energy and the total energy that accumulates during sticking (Eq. (13)). Specifically, the energy transition can be represented by two important points: the slip starting point $x_{\mathrm{s}}$ and the slip ending point $x_{\mathrm{e}}$ of the oscillator. The spring stiffness determines how much energy is invested in the system during the stick stage, as proposed by Krylov and Frenken in a recent review paper [32]. Generally, less potential is stored in the case of a stiffer spring. Moreover, the stiffness $k$ can influence the EDR by affecting the slipping distance. In order to explain this clearly, three oscillator trajectories are shown in Fig. 6 , with $k=3,6$, and $9 \mathrm{~N} / \mathrm{m}$, respectively. In these 


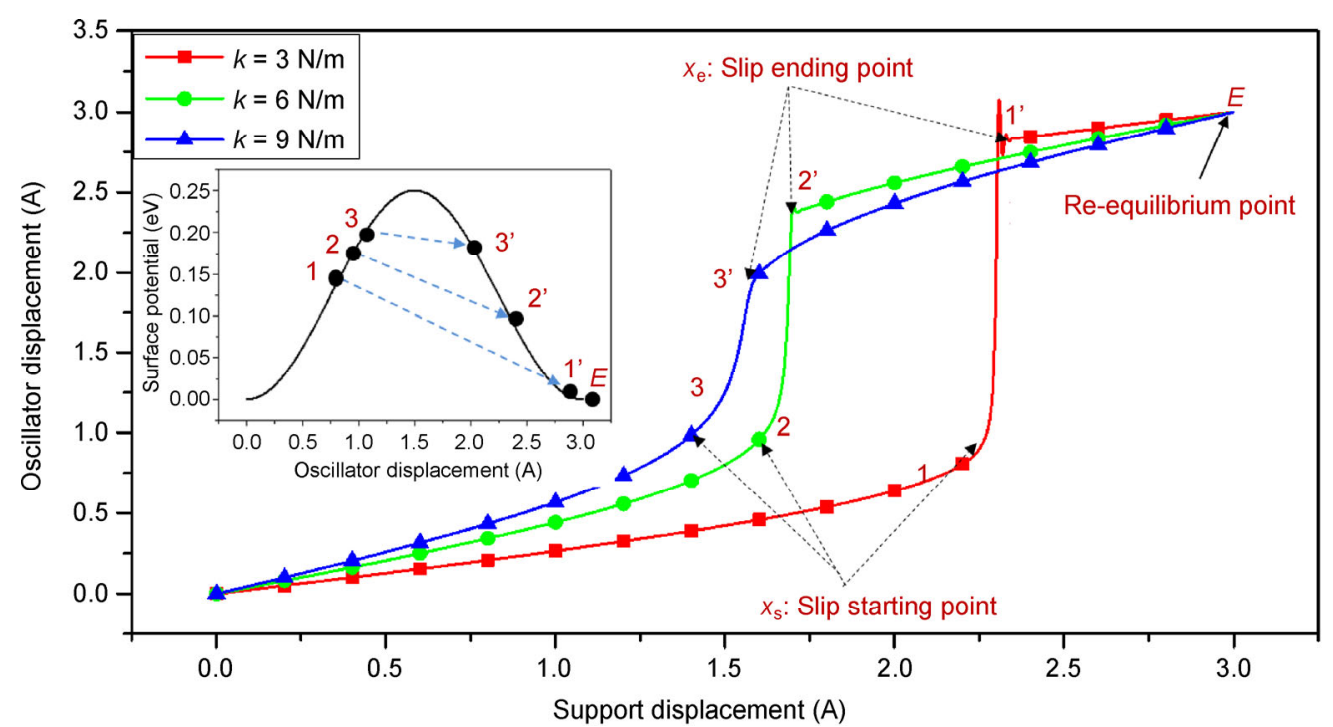

Fig. 6 Three trajectories with stiffness values of 3, 6, and $9 \mathrm{~N} / \mathrm{m}$, respectively. Points 1, 2, and 3 are the slip-starting points under different stiffness values, and points $1^{\prime}, 2^{\prime}, 3^{\prime}$ are the corresponding slip-ending points. The simulation is conducted under the following conditions: damping constant $c=0.6, \mathrm{~T}=0 \mathrm{~K}, U=0.25 \mathrm{eV}$. The inset shows the surface potential in a lattice period, where the slip starting and ending points are marked.

three cases, the $x_{\mathrm{s}}$ positions of the oscillator are relatively close to each other (labeled as 1, 2, 3), and the slipping distance (from 1-1', 2-2', and 3-3', respectively) decreases as the spring stiffness increases. The slip ending point moves continuously from the peak point to the valley point of the surface potential, as shown in the inset of Fig. 6, which explains the continuous variation of the EDR from $100 \%$ to 0 . Consequently, the $x_{\mathrm{e}}$ positions are given by the coordinates of 2.8, 2.3, and $1.9 \AA$ (denoted as $1,2^{\prime}$, and $3^{\prime}$, respectively). These points correspond to surface potential energies of $0.011,0.112$, and $0.208 \mathrm{eV}$, as indicated by the Fig. 6 inset and Table 1. According to the definition shown in Fig. 3, this potential energy is exactly equal to the $\mathrm{RP}_{\mathrm{s}}$ and is released from point $1^{\prime}$ (or 2', 3') to the re-equilibrium point $(E)$. When the stiffness varies from 3 to $9 \mathrm{~N} / \mathrm{m}$, the $\mathrm{RP}_{\mathrm{s}}$ varies by a factor greater than 20 and plays an important role in determining the amount of reversible energy. Thus, the $\mathrm{RP}_{\mathrm{s}}$ greatly influences the EDR values listed in Table 1. Consequently, the friction dissipation depends not only on the amount of energy invested in the system but also, more importantly, on the amount of reversible energy. The stiffness can greatly influence the slipping distance and thus the amount of $\mathrm{RP}_{\mathrm{s}}$ which plays an important role in determining the amount of reversible energy.
Table 1 EDR values under different spring-stiffness conditions. The simulation is conducted under the conditions indicated in Fig. 6.

\begin{tabular}{cccccc}
\hline $\begin{array}{c}\text { Spring } \\
\text { stiffness } k \\
(\mathrm{~N} / \mathrm{m})\end{array}$ & $\begin{array}{c}\text { Slip ending } \\
\text { position }(\AA)\end{array}$ & $\begin{array}{c}\mathrm{RP}_{\mathrm{s}} \\
(\mathrm{eV})\end{array}$ & $\begin{array}{c}\mathrm{RP}_{\mathrm{e}} \\
(\mathrm{eV})\end{array}$ & $\begin{array}{c}\text { Energy } \\
\text { peak value } \\
(\mathrm{eV})\end{array}$ & $\mathrm{EDR}$ \\
\hline 3 & 2.8 & 0.011 & 0.024 & 0.345 & $89.97 \%$ \\
6 & 2.3 & 0.112 & 0.058 & 0.277 & $38.60 \%$ \\
9 & 1.9 & 0.208 & 0.030 & 0.258 & $7.64 \%$ \\
\hline
\end{tabular}

\subsection{Influence of damping coefficient}

According to the Langevin equation, damping causes energy dissipation. Thus, it is straightforward to discuss the influence of the damping coefficient. We used a damping constant $c$ ranging from 0.6 to 2.0 to ensure a single slip during the sliding, while covering all the under-damping, critical-damping, and overdamping states. As shown in Fig. 7, the EDR increases slightly as the damping coefficient increases. The influence of the damping coefficient is less than $10 \%$. Nonetheless, it is inappropriate to conclude that the damping coefficient has a small effect on the EDR, although it is the case under this specific condition $\left(v_{\mathrm{DR}}=1 \mu \mathrm{m} / \mathrm{s}, \mathrm{T}=0 \mathrm{~K}\right)$.

Nevertheless, the damping coefficient profoundly impacts how the energy dissipates. In Fig. 2, we divide the whole stick-slip period into four stages with 


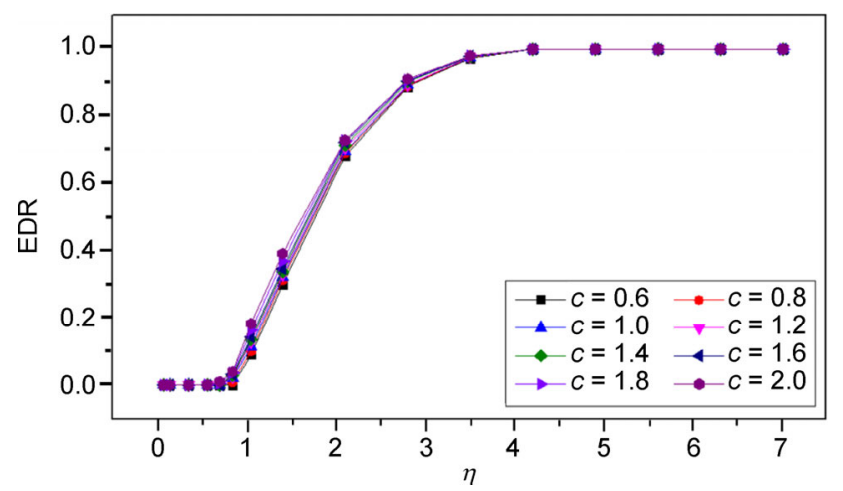

Fig. 7 EDR curves of various $\eta$ values with different damping constants. The damping coefficient increases from 0.6 to 2.0, which covers all the three states: under-damping, critical damping, and over-damping. This series of simulations is performed under $v_{\mathrm{DR}}=$ $1 \mu \mathrm{m} / \mathrm{s}$.

regard to the energy transitions. Correspondingly, from the viewpoint of dynamics, these can be regarded as the stick, primary-slip, oscillation, and re-equilibrium stages, as shown in Fig. 8. Considering $\eta=8.8$ (thus, EDR value is $100 \%$ ) as an example, different damping coefficients lead to completely different results with regard to both the dynamics and energy dissipation, as shown in Fig. 8. In the case of low damping $(c=0.6)$, a strong and long-lived oscillation stage is observed at the end of the primary slip, which accounts for $\sim 22 \%$ of the total dissipation energy (Fig. 8(a)). However, in the case of high damping $(c=2.0)$, there is almost no oscillation, and over $99 \%$ of the energy dissipation occurs in the primary slip stage (Fig. 8(b)). Apart from this, the damping coefficient greatly influences the speed of energy dissipation. As shown in Fig. 8, the slip stage lasts 3 and $11 \mu$ s with high and low damping coefficients, respectively. Hence, compared with its less obvious effect on the EDR, the damping coefficient has a remarkable influence on the system dynamics. A system with a high damping coefficient can quickly achieve its stable state.

Energy dissipation is a complex process involving various pathways. The stick-slip phenomenon is an important cause of the energy dissipation in atomicscale friction. The sudden slip and oscillation result in lattice vibrations and phononic dissipation, and phonons are the quantum representation of the lattice vibration energy [33]. The collisions among phonons introduce inharmonic acoustic modes, which can be rapidly converted to heat. The lattice vibrations can also be transformed into acoustic waves or luminous flashes rather than coupling with the heat generation.
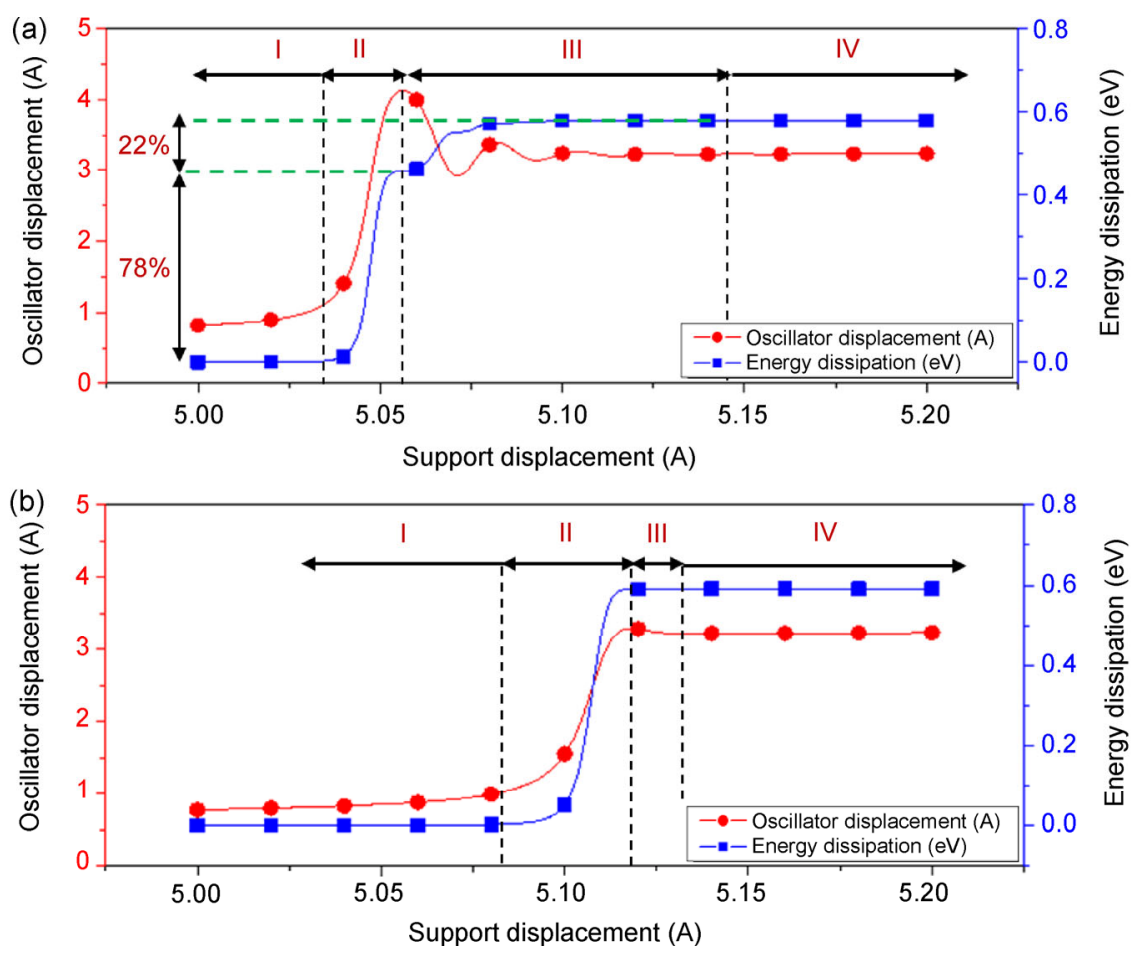

Fig. 8 Dynamical influence of the damping coefficient. Curves of the oscillator displacement and energy dissipation are plotted for (a) damping constant $c=0.6$; (b) damping constant $c=2.0$. Other parameters: $U=0.25 \mathrm{eV}, k=1 \mathrm{~N} / \mathrm{m}$ (thus, $\eta=8.8$ ), $v_{\mathrm{DR}}=1 \mu \mathrm{m} / \mathrm{s}$. 
Moreover, recent studies indicate that energy can be dissipated in the stick stage as well, which is attributed to thermoelastic damping [34-36]. Various factors, including the humidity, viscosity, and temperature, may contribute crucially to the damping coefficient.

\subsection{Influence of temperature}

The thermal effect on atomic-scale friction have been reported for years $[10,29,32,37,38]$. One of the explanations is that random fluctuation provides extra energy input and helps the oscillator surpass the energy barrier, thus reducing the mean friction and even achieving a superlow friction [32]. Here, we provide a detailed analysis of the thermal effect on atomic-scale stick-slip motion with regard to the energy dissipation.

The Langevin equation for describing the PT model comprises both deterministic dynamics and stochastic
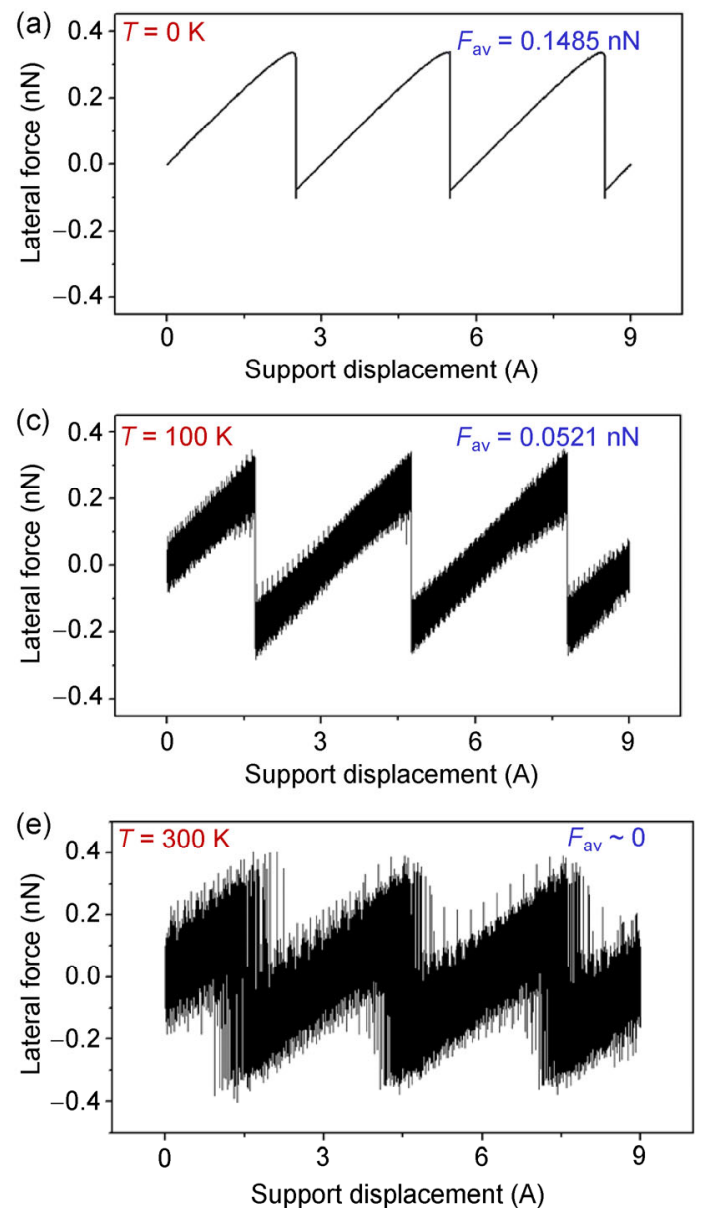

processes. Thermal fluctuation is a common stochastic process that is frequently used in dynamic simulations. In this paper, the thermal fluctuation is represented by a random force $\varepsilon(t)$ that follows the fluctuationdissipation relation, as shown in Eq. (15). In this expression, the mean value of the autocorrelation function of the thermal force is used to measure the randomness of the thermal effect. $k_{\mathrm{B}}$ is the Boltzmann constant, and $\delta$ indicates the Dirac delta function. Equation (15) indicates that $\varepsilon(t)$ is a random term whose the average amplitude is proportional to $\sqrt{T}$ :

$$
\left\langle\varepsilon(t) \varepsilon\left(t^{\prime}\right)\right\rangle=2 m \mu k_{\mathrm{B}} T \delta\left(t-t^{\prime}\right)
$$

To investigate the thermal effect, the friction and system potentials at three temperatures $-0,100$, and $300 \mathrm{~K}$-are compared in Fig. 9. It is immediately apparent that a higher temperature yields more intense fluctuations. The average lateral force $\left(F_{\mathrm{av}}\right)$
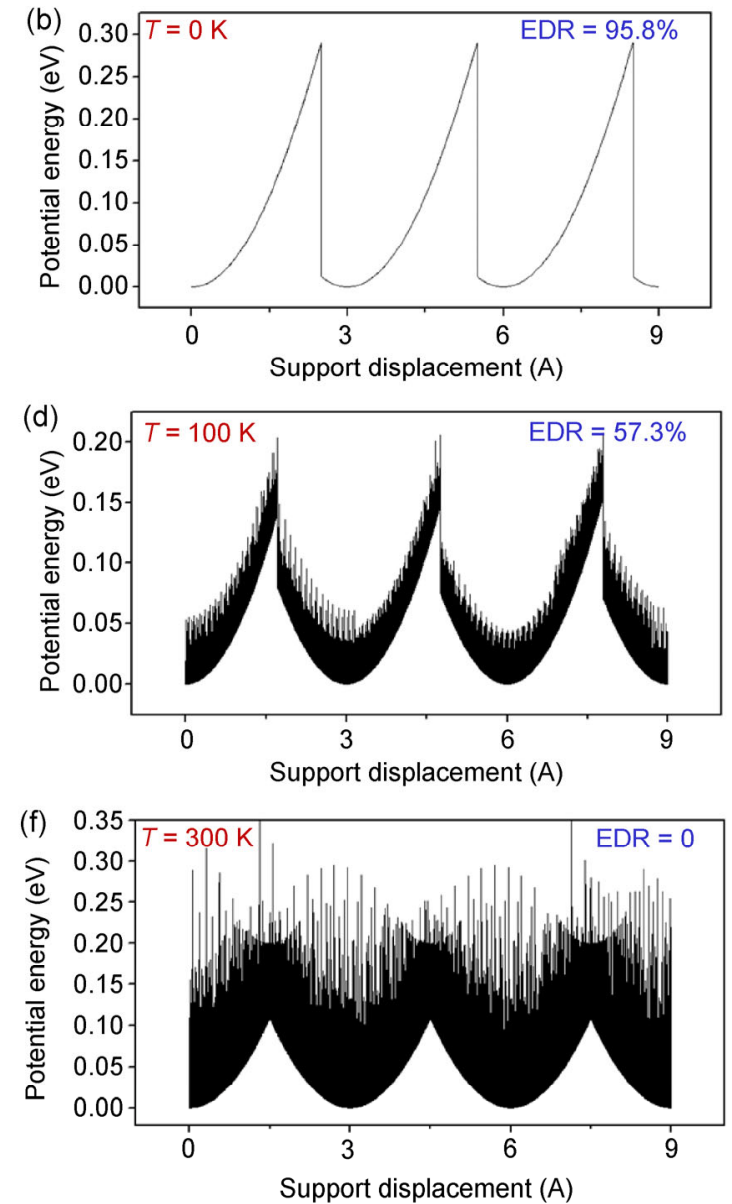

Fig. 9 Influence of the temperature on the EDR. (a), (c), and (e) Lateral-force variation at temperatures of 0, 100, and 300 K, respectively. (b), (d), and (f) Variations of system potential energy during sliding. Other parameters: $k=2 \mathrm{~N} / \mathrm{m}, U=0.2 \mathrm{eV}, c=1.0, v_{\mathrm{DR}}=0.01 \mu \mathrm{m} / \mathrm{s}$. 
decreases rapidly with the temperature. As shown in Figs. 9(a), 9(c), and 9(e), $F_{\text {av }}$ is $0.1485,0.0521$, and nearly $0 \mathrm{nN}$. With regard to the energy, a thermally induced fluctuation can greatly increase the probability of the oscillator jumping across the energy barrier. This reduces the potential-energy accumulation during the stick stage, which decreases from 0.29 at $0 \mathrm{~K}$ to $0.18 \mathrm{eV}$ and $0.12 \mathrm{eV}$ at 100 and $300 \mathrm{~K}$, as indicated by the lower profiles of the curves shown in Figs. 9(b), 9(d), and 9(f), respectively. This is similar to the previously proposed thermolubricity mechanism. The proportion of the reversible energy (RP) in the slip stage also increases with the temperature, as estimated by the potential energy curves. With the combined effects of decreasing the energy accumulation and increasing the energy reversibility, the EDR exhibits a remarkable decrease with the temperature $(95.8 \%, 57.3 \%$, and nearly 0 at 0,100 , and $300 \mathrm{~K}$, respectively). In an extreme case, superlow friction can be achieved at $300 \mathrm{~K}$, as shown in Figs. 9(e) and 9(f). This superlubricity regime should be distinguished from the smoothsliding regime as described in Section 3.2, where the system is stable with a very low $\eta$ value. In this case, the thermal fluctuation is sufficiently strong to enable the "forward jump" and "backward jump" [32] across the energy barrier. In this superlubricity regime, the stick-slip instability remains, and the major reason for the extremely small energy dissipation is the reversibility of the mechanical energy that accumulates in the stick stage during the slipping.

To understand the synergetic effects of $\eta$ and the thermal effect on the friction energy dissipation, $\eta-\mathrm{T}$ phase diagrams of the EDR are drawn for driving velocities of $v_{D R}=1 \mu \mathrm{m} / \mathrm{s}$ (Fig. 10(a)) and $0.01 \mu \mathrm{m} / \mathrm{s}$ (Fig. 10(b)). In both graphs, two white curves indicate the boundaries of the regions for $\mathrm{EDR}=100 \%$ and 0 . For a fixed temperature, the variation of the EDR as $\eta$ decreases is similar to the curve shown in Fig. 4. This phase diagram suggests two possible pathways to achieve superlubricity, as indicated by the arrows in Fig. 10: by increasing the system stability (one example is the structural lubricity with a very low potential-energy corrugation) and by increasing the temperature to reach a state of thermolubricity. However, both mechanisms are actually unified with regard to the energy dissipation and can be regarded
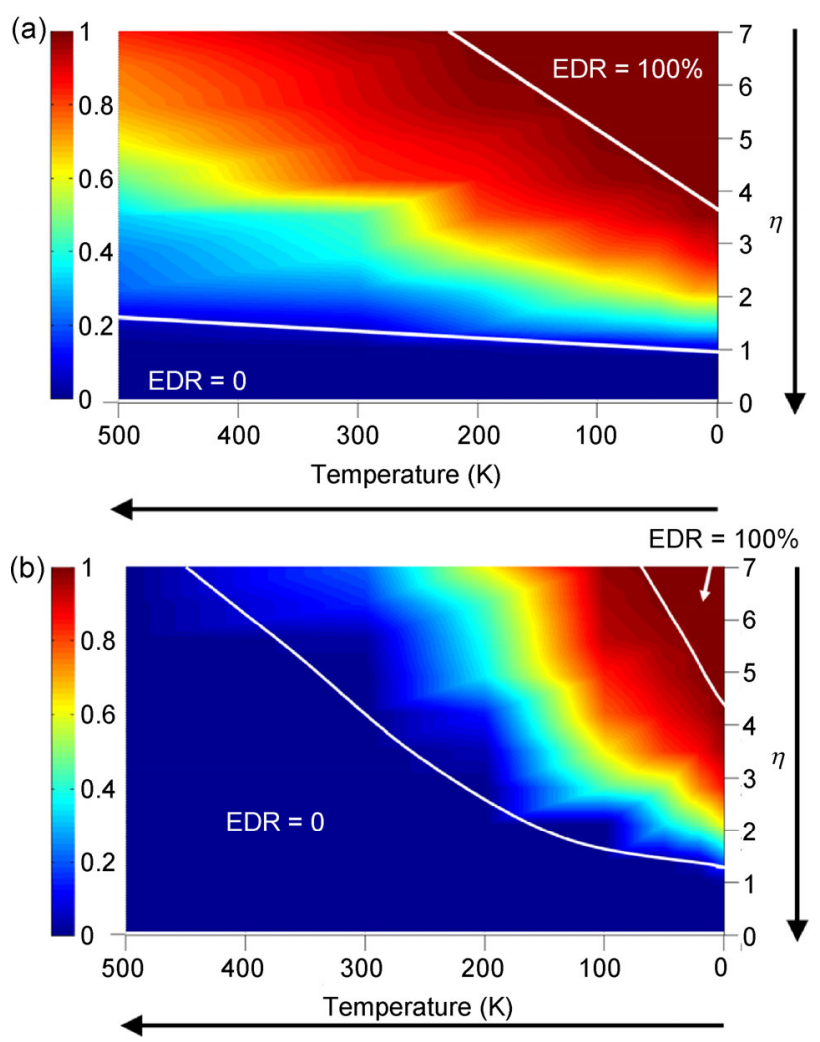

Fig. 10 EDR graphs with $\eta$ (vertical axis) and temperatures (horizontal axis) under $v_{\mathrm{DR}}$ of (a) $1 \mu \mathrm{m} / \mathrm{s}$ and (b) $0.01 \mu \mathrm{m} / \mathrm{s}$. Two solid curves mark the limits of EDR $=100 \%$ and 0 . Two solid arrows indicate the two possible pathways to achieve superlubricity: decreasing $\eta$ and increasing the temperature.

as energy-reversible superlubricity, as the EDR is mainly determined by the proportion of the reversible energy after slipping. It should be mentioned that the sliding velocity also has important effects on the friction dissipation. This was systematically reviewed by Krylov and Frenken [32] and is not discussed in the present paper.

\subsection{Analytical formulation of EDR}

In the previous sections, the EDR is calculated according to the energy-transition process, which is difficult to measure in real experimental systems. To validate our theoretical prediction, an analytical formulation of the EDR is derived directly from the lateral-force curve shown in Fig. 11(a). Here, we use the definition of $\mathrm{EDR}_{W}$ shown in Eq. (12) for the comparison with the experimental force curves. For simplicity, the lateral force is approximated by a perfect sawtooth curve. In Fig. 3(a), the area enclosed by the positive lateral 

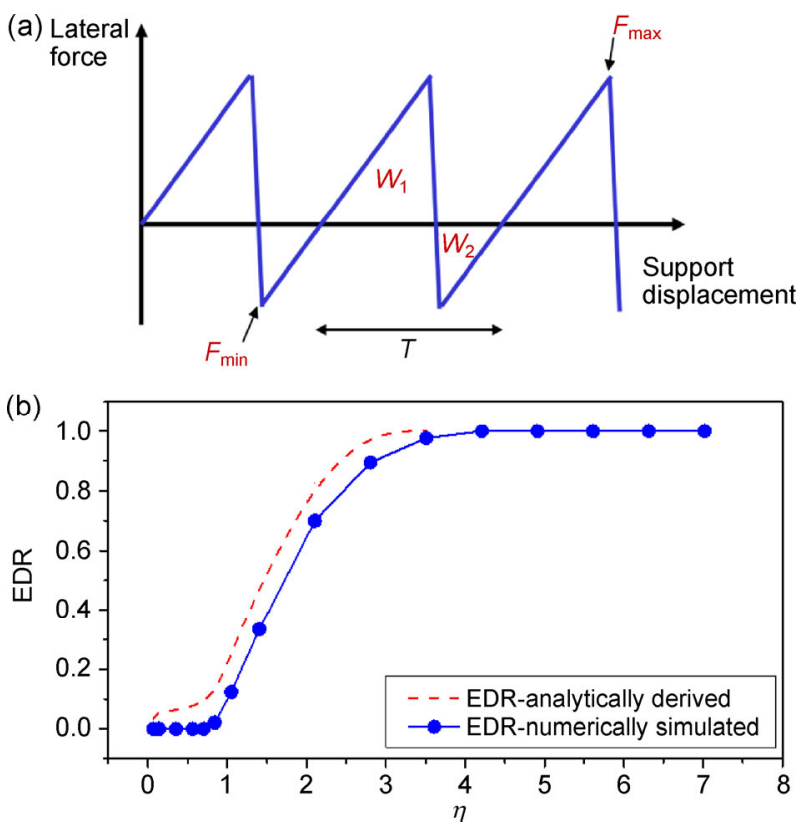

Fig. 11 Analytical formulation of EDR. (a) Schematic curve of lateral force in atomic-scale stick-slip. In a stick-slip period, the area enclosed by the positive-force curve and the horizontal axis is $W_{1}$, which indicates the positive work done by the external force to the system. $W_{2}$ indicates the work transferred to the external system. (b) Comparison of the analytically derived results (according to Eq. (16)) and numerically simulated results for the EDR. Other parameters: $v_{\mathrm{DR}}=1 \mu \mathrm{m} / \mathrm{s}, T=0 \mathrm{~K}, c=1.0$.

force $W_{1}$ indicates positive work done by the external system during a stick-slip period. Meanwhile, the area enclosed by the negative lateral force $W_{2}$ indicates the energy retrieval from the internal system.

Considering the geometry of the lateral-force curve, Eq. (12) can be rewritten as Eq. (16), where $S$ is the slip length of the oscillator $\left(S=x_{\mathrm{e}}-x_{\mathrm{s}}\right)$ :

$$
\begin{aligned}
& \mathrm{EDR}=\left\{\begin{array}{l}
1 \\
\frac{F_{\text {max }}^{2}-F_{\text {min }}^{2}}{F_{\text {max }}^{2}}=\frac{\left(F_{\text {max }}+F_{\text {min }}\right)\left(F_{\text {max }}-F_{\text {min }}\right)}{(\pi U / a)^{2}}=\frac{4 F_{\mathrm{av}} S}{\eta U}
\end{array}\right. \\
& \text { If } \mid \begin{array}{l}
F_{\text {min }} \geqslant 0 \\
F_{\text {min }}<0
\end{array} \\
& \mathrm{EDR}=\left\{\begin{array}{l}
1 \\
\frac{F_{\text {max }}^{2}-F_{\text {min }}^{2}}{F_{\text {max }}^{2}}=\frac{(k L)^{2}-(k L-k S)^{2}}{(k L)^{2}}=2 \alpha(1-\alpha / 2)
\end{array}\right. \\
& \text { If } \begin{array}{l}
\alpha \leqslant 1 \\
1<\alpha<2
\end{array}
\end{aligned}
$$

In Eq. (16), $\eta$ and $U$ are intrinsic system parameters, while $F_{\text {av }}$ and $S$ are two variables that depend on various parameters, such as the damping coefficient, temperature, and particularly the $\eta$ value. Figure 11(b) compares the analytical results and numerical simulated results for the EDR. The two curves exhibit highly similar tendencies, although there are small differences because the lateral-force curve does not have a perfect sawtooth shape, as assumed in the analytical derivation, but contains some irregularities and distortions. For example, sometimes $F_{\max }+F_{\min }<$ $2 F_{\text {av }}$ because of dynamic effects such as damping. Thus, Eq. (16) behaves better under the conditions of low damping and a low sliding velocity. Nonetheless, Eq. (16) demonstrates the consistency and relationship between the lateral force and energy dissipation in atomic-scale friction. More importantly, the proposed method is applicable for the prediction of atomicscale friction in real AFM or friction force microscopy (FFM) experiments [39].

The expression of the EDR can be further simplified (Eq. (17)) by defining a dimensionless parameter $\alpha=S / L$, where $\mathrm{L}$ is the maximum elongation of the spring, and $\alpha$ can be viewed as the degree of recovery of the spring elongation during slipping. $\alpha \leqslant 1$ indicates the partial or complete recovery of the spring deformation. $1<\alpha<2$ indicates the over-recovery of the spring deformation or over-slipping of the oscillator, which causes energy reversibility. Although $\alpha$ is dependent on various system factors such as $\eta, \mu$, T, and $v_{\mathrm{DR}}$, this expression provides an intuitive understanding of the energy dissipation according to the system sliding behaviors (slip distance and maximum elongation of spring).

\section{Conclusion}

In this study, atomic-scale friction is examined with regard to energy, and the energy dissipation and transition during sliding are examined systematically. First, a reversible energy exchange between internal and external systems is observed under atomic friction, and the concept of the EDR is proposed to measure the degree of energy reversibility. The spring stiffness and surface potential, including the comprehensive parameter $\eta$, are the main factors influencing the EDR. We observe a continuous variation of the EDR 
from $100 \%$ to 0 with $\eta$ covering the stick-slip, intermediate, and smooth-sliding regimes. For a sufficiently small $\eta$, smooth-sliding superlubricity is achieved, and no energy is dissipated. Furthermore, both the damping coefficient and temperature are found to impact the amount and dynamics of energy dissipation. In particular, the thermal effect tends to decrease the potential energy accumulation during sticking and increase the proportion of reversible energy during slipping, yielding a state of thermolubricity. On the basis of $\eta-T$ phase diagrams of the EDR, we propose that the smooth-sliding superlubricity and thermolubricity are actually unified with regard to the energy dissipation and transition. Finally, an analytical formulation of the EDR is derived. This not only deepens our understanding of the energy dissipation during atomic-scale friction but also is applicable for judging energy-dissipation properties for real AFM/FFM experiments and provides criteria for saving energy in the design and fabrication of micro-frictional systems.

\section{Acknowledgments}

We thank Prof. Ashlie Martini and Dr. Yalin Dong for helpful discussions. This work is financially supported by the National Natural Science Foundation of China (Grant Nos. 51422504, 51375010, and 51371092) and the National Key Basic Research (973) Program of China (No. 2013CB934200). Computations were carried out on the "Explorer 100" cluster system of Tsinghua National Laboratory for Information Science and Technology.

Open Access: This article is distributed under the terms of the Creative Commons Attribution License which permits any use, distribution, and reproduction in any medium, provided the original author(s) and source are credited.

\section{References}

[1] Muser M H. Nature of mechanical instabilities and their effect on kinetic friction. Phys Rev Lett 89(22): 224301 (2002)

[2] Park J Y, Salmeron M. Fundamental aspects of energy dissipation in friction. Chem Rev 114(1): 677-711 (2014)

[3] $\mathrm{Hu}$ Y Z, Ma T B, Wang H. Energy dissipation in atomicscale friction. Friction 1(1): 24-40 (2013)
[4] Singer I L. Friction and energy dissipation at the atomic scale: A review. J Vac Sci Technol A 12(5): 2605 (1994)

[5] Colchero J, Baro A M, Marti O. Energy dissipation in scanning force microscopy-friction on an atomic scale. Tribol Lett 2(4): 327-343 (1996)

[6] Johnson K L, Woodhouse J. Stick-slip motion in the atomic force microscope. Tribol Lett 5(2-3): 155-160 (1998)

[7] Berman A D, Israelachvili J N. Modern Tribology Handbook. CRC Press, 2000.

[8] Socoliuc A, Bennewitz R, Gnecco E, Meyer E. Transition from stick-slip to continuous sliding in atomic friction: Entering a new regime of ultralow friction. Phys Rev Lett 92(13): 134301 (2004)

[9] Rozman M G, Urbakh M, Klafter J. Origin of stick-slip motion in a driven two-wave potential. Phys Rev E 54(6): 64856494 (1996)

[10] Prandtl L. Ein Gedankenmodell zur kinetischen theorie der festen Körper. Z Angew Math Mech 8: 85-106 (1928)

[11] Tomlinson G A. A molecular theory of friction. Phil Mag 7(46): 905 (1929)

[12] Popov V L, Gray J A T. In Prandtl-Tomlinson Model: A Simple Model Which Made History: The History of Theoretical, Material and Computational Mechanics-Mathematics Meets Mechanics and Engineering. Springer Berlin Heidelberg, 2014: 153-168.

[13] Hirano M. Superlubricity: A state of vanishing friction. Wear 254(10): 932-940 (2003)

[14] Hirano M. Atomistics of superlubricity. Friction 2(2): 95-105 (2014)

[15] Meyer E, Gnecco E. Superlubricity on the nanometer scale. Friction 2(2): 106-113 (2014)

[16] Frenkel J, Kontorova T. On the theory of plastic deformation and twinning. Zh Eksp Teor Fiz 8: 1340 (1938)

[17] Peyrard M, Aubry S. Critical-behavior at the transition by breaking of analyticity in the discrete Frenkel-Kontorova model. J Phys C 16(9): 1593 (1983)

[18] Weiss M, Elmer F J. Dry friction in the FrenkenKontorova-Tomlinson model: Static properties. Phys Rev B 53: 7539 (1996)

[19] Xu Z M, Huang P. Study on the energy dissipation mechanism of atomic-scale friction with composite oscillator model. Wear 262(7-8): 972-977 (2007)

[20] Dong Y L, Vadakkepatt A, Martini A. Analytical models for atomic friction. Tribol Lett 44(3): 367-386 (2011)

[21] Zwanzig R. Nonlinear generalized Langevin equations. $J$ Stat Phys 9: 215 (1973)

[22] Ullersma P. An exactly solvable model for Brownian motion: IV. Susceptibility and Nyquist's theorem. Physica 32(1): 90-96 (1966)

[23] Tománek D, Zhong W, Thomas H. Limits of resolution in 
atomic force microscopy images of graphite. Europhys Lett 15(49): 887 (1991)

[24] Steiner P, Roth R, Gnecco E, Baratoff A, Maier S, Glatzel T, Meyer E. Two-dimensional simulation of superlubricity on $\mathrm{NaCl}$ and highly oriented pyrolytic graphite. Phys Rev B 79(4): 045414 (2009)

[25] Roth R, Glatzel T, Steiner P, Gnecco E, Baratoff A, Meyer E. Multiple slips in atomic-scale friction: An indicator for the lateral contact damping. Tribol Lett 39(1): 63-69 (2010)

[26] Lyapunov A M. The general problem of the stability of motion. Ph.D Thesis. Moscow: 1892.

[27] Barrett J F. Bibliography of A.M. Lyapunov's work. Int J Control 55: 785-790 (1992)

[28] Verhoeven G S, Dienwiebel M, Frenken J W M. Model calculations of superlubricity of graphite. Phys Rev B 70(16): 165418 (2004)

[29] Krylov S Y, Jinesh K B, Valk H, Dienwiebel M, Frenken J W. Thermally induced suppression of friction at the atomic scale. Phys Rev E 71(6 Pt 2): 65101 (2005)

[30] Medyanik S N, Liu W K, Sung I H, Carpick R W. Predictions and observations of multiple slip modes in atomic-scale friction. Phys Rev Lett 97(13): 136106 (2006)

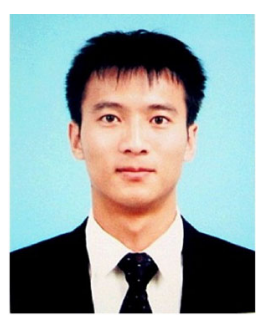

Zijian WANG. He received his bachelor degree in mechanical engineering in 2013 from Tsinghua University, Beijing, China. During his undergraduate, he joined a double-degree program and studied

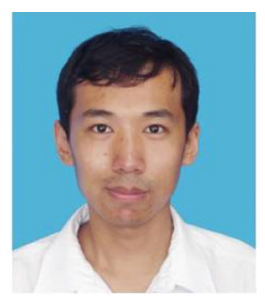

Tianbao MA. He received his Ph.D degree in mechanical engineering in 2007 from Tsinghua University, Beijing, China. He joined the State Key Laboratory of Tribology at

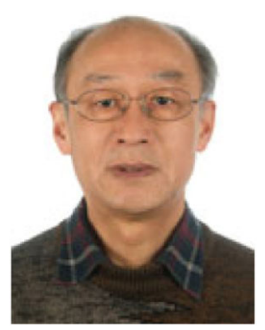

Yuanzhong HU. He graduated in 1968 from Tsinghua University, and received his $\mathrm{PhD}$ in 1985. Since then he joined the State Key Laboratory of Tribology, Tsinghua University, as an associate professor and later a professor. During 19891992, he worked in Norwegian
[31] Xu L, Ma T B, Hu Y Z, Wang H. Vanishing stick-slip friction in few-layer graphenes: The thickness effect. Nanotechnology 22(28): 285708 (2011)

[32] Krylov S Y, Frenken J W M. The physics of atomic-scale friction: Basic considerations and open questions. Physica Status Solidi (B) 251(4): 711-736 (2014)

[33] Huang K, Han R Q. Solid State Physics. China Higher Education Press, 1988.

[34] Zener C. General theory of thermoelastic internal friction. Phys Rev 53: 90 (1938)

[35] Lifshitz R, Roukes M L. Thermoelastic damping in microand nanomechanical systems. Phys Rev B 61(8): 5600 (2000)

[36] Klein J. Frictional dissipation in stick-slip sliding. Phys Rev Lett 98(5): 56101 (2007)

[37] Krylov S Y, Frenken J W M. The crucial role of temperature in atomic scale friction. $J$ Phys: Condens Matter 20(35): 354003 (2008)

[38] Sang Y, Dube M, Grant M. Thermal effects on atomic friction. Phys Rev Lett 87(17): 174301 (2001)

[39] Lee C, Li Q Y, Kalb W, Liu X Z, Berger H, Carpick R W, Hone J. Frictional characteristics of atomically thin sheets. Science 328: 76 (2010)

in Ecole Centrale de Lyon, France. He is currently a M.S. student in the State Key Laboratory of Tribology at Tsinghua University. His research interests include energy dissipation in friction, lattice vibrations and phonon emission induced by friction.

Tsinghua University at 2009. His current position is an associate professor. His research area covers the atomic-scale friction, superlubricity, tribochemistry and nanomanufacturing.

Technical University, Norway, and in Northwestern University, US, as a postdoctoral fellow. He studied in the US again during 1997.5-1998.12, as a visiting scholar. His research interests include EHL, mixed lubrication, wear dynamics, nano-tribology, thin film rheology, molecular dynamics simulations, etc., and in these areas he has more than 100 papers published on international journals. 